\title{
A SPECIALITY THEOREM FOR CURVES IN $\mathbf{P}^{5}$
}

\author{
Vincenzo Di Gennaro* and Davide Franco**
}

\begin{abstract}
Let $C \subset \mathbf{P}^{r}$ be an integral projective curve. One defines the speciality index $e(C)$ of $C$ as the maximal integer $t$ such that $h^{0}\left(C, \omega_{C}(-t)\right)>0$, where $\omega_{C}$ denotes the dualizing sheaf of $C$. Extending a classical result of Halphen concerning the speciality of a space curve, in the present paper we prove that if $C \subset \mathbf{P}^{5}$ is an integral degree $d$ curve not contained in any surface of degree $<s$, in any threefold of degree $<t$, and in any fourfold of degree $\left\langle u\right.$, and if $d>>s>>t>>u \geq 1$, then $e(C) \leq \frac{d}{s}+\frac{s}{t}+\frac{t}{u}+u-6$. Moreover equality holds if and only if $C$ is a complete intersection of hypersurfaces of degrees $u, \frac{t}{u}, \frac{s}{t}$ and $\frac{d}{s}$. We give also some partial results in the general case $C \subset \mathbf{P}^{r}$, $r \geq 3$.
\end{abstract}

Keywords and phrases: Complex projective curve, speciality index, arithmetic genus, adjunction formula, complete intersection, linkage, Castelnuovo - Halphen Theory, flag conditions.

Mathematics Subject Classification 2000: Primary 14N15, 14H99, 14M10; Secondary 14M06, $14 \mathrm{~N} 30$.

Let $C \subset \mathbf{P}^{r}$ be an integral projective curve. One defines the speciality index $e(C)$ of $C$ as the maximal integer $t$ such that $h^{0}\left(C, \omega_{C}(-t)\right)>0$, where $\omega_{C}$ denotes the dualizing sheaf of $C$. In [GP] Gruson and Peskine prove the following theorem concerning the speciality index of an integral space curve:

Theorem A. Let $C \subset \mathbf{P}^{3}$ be an integral degree $d$ curve not contained in any surface of degree $<s$. Then one has:

$$
e(C) \leq \frac{d}{s}+s-4
$$

Moreover equality holds if and only if $C$ is a complete intersection of surfaces of degrees $s$ and $\frac{d}{s}$.

In the present paper we extend this theorem to curves in $\mathbf{P}^{5}$, in the following sense:

\footnotetext{
*Dipartimento di Matematica, Università di Roma "Tor Vergata", 00133 Roma, Italy. E-mail: digennar@axp.mat.uniroma2.it

**Dipartimento di Matematica e Applicazioni "R. Caccioppoli", Università di Napoli "Federico II", Ple Tecchio 80, 80125 Napoli, Italy. E-mail: dfranco@unina.it
} 
Theorem B. Let $C \subset \mathbf{P}^{5}$ be an integral degree d curve not contained in any surface of degree $<s$, in any threefold of degree $<t$, and in any fourfold of degree $<u$. Assume $d>>s>t>>\geq 1$. Then one has:

$$
e(C) \leq \frac{d}{s}+\frac{s}{t}+\frac{t}{u}+u-6
$$

Moreover equality holds if and only if $C$ is a complete intersection of hypersurfaces of degrees $u, \frac{t}{u}, \frac{s}{t}$ and $\frac{d}{s}$.

In Remark $(i)$ below, we make explicit the condition $d>>s>>t>>u$.

Taking into account the general inequality:

$$
\operatorname{deg}(C) \cdot e(C) \leq 2 p_{a}(C)-2
$$

$\left(p_{a}(C)=\right.$ arithmetic genus of $\left.C\right)$, in [GP], Remarque 3.6, the authors notice that Theorem $\mathrm{A}$ is an immediate consequence of the Halphen bound for the genus of space curves and the consequent classification of the curves with maximal genus. Similarly, as we will see in the proof, in the case $C \subset \mathbf{P}^{4}$, i.e. when $u=1$, our Theorem $\mathrm{B}$ is an easy consequence of the analogue of the Halphen bound for the genus of curves in $\mathbf{P}^{4}$ verifying flag conditions, shown in [CCD].

Given integers $s_{1}, s_{2}, \ldots, s_{l}$, with $1 \leq l \leq r-1$ and $s_{l} \geq r-l+1$, we say that a nondegenerate integral projective curve $C \subset \mathbf{P}^{r}$ verifies the flag condition $\left(r ; s_{1}, s_{2}, \ldots, s_{l}\right)$ if $C$ has degree $s_{1}$, and, for any $i=2, \ldots, l$, it is not contained in any integral, projective subvariety of $\mathbf{P}^{r}$ of dimension $i$ and degree $<s_{i}$. Except for the case $l \leq 2$ (see [CCD3]), for the case $l=3$ and $r=4$ (see [CCD]), and for some partial results in the case $l \geq 3$ (see [CCD2]), it is not known an analogue of the Halphen bound for curves verifying flag conditions in $\mathbf{P}^{r}, r \geq 5$. Therefore one cannot obtain a speciality theorem in $\mathbf{P}^{r}$, $r \geq 5$, as a direct consequence of Halphen bound as indicated before.

In the case $r=5$ we are able to overcome this difficulty in the following way. First we notice that, under the hypotheses of Theorem $\mathrm{B}$, from (1) it follows that if $e(C) \geq$ $\frac{d}{s}+\frac{s}{t}+\frac{t}{u}+u-6$, then the arithmetic genus of $C$ is "sufficiently big" to apply [CCD2], Theorem 2.2, and so to deduce that $C$ belongs to a flag such as

$$
C \subset S \subset T \subset U \subset \mathbf{P}^{5},
$$

where $S, T$ and $U$ are a surface, a threefold and a fourfold of degrees $s, t$ and $u$. Next, using a sort of a numerical coarse adjunction formula on $S$ (see Lemma 1 below), we are able to compare the sectional genus of $S$ with the Halphen bound for curves in $\mathbf{P}^{4}$ quoted before, and to deduce that $S$ is in fact a complete intersection (for the proof of Lemma 1, which relies on Castelnuovo Theory, we refer to [D]; see also Remark (ii) below for further comments). At this point Theorem B follows from Lemma 2 below, which is a generalized "weak" version of Theorem A, for curves contained in a complete intersection surface. The proof of Lemma 2 relies on a liaison argument, and does not need the numerical assumption on the degrees.

Since the proof of Theorem B depends on results obtained in [CCD], [CCD2] and [D], we are forced to assume that $r=5$, and that $d>>s>>t>>u$. We use this last condition also in some numerical estimates: see (8), (9) and (13) below.

In the general case concerning curves in $\mathbf{P}^{r}, r \geq 3$ and $1 \leq l \leq r-1$, we notice the following partial result. 
Proposition 1. Let $C \subset \mathbf{P}^{r}$ be a reduced and irreducible curve verifying the flag condition $\left(r ; s_{1}, \ldots, s_{l}\right)$. Assume that $s_{1}>>s_{2}>>\cdots>>s_{l}$. Then one has:

(i)

$$
e(C) \leq \frac{s_{1}}{s_{2}}+\frac{s_{2}}{s_{3}}+\cdots+\frac{s_{l}}{s_{l+1}}-l-\frac{2}{r-l}+\frac{3}{4} ;
$$

in particular when $l=r-1$ then

$$
e(C) \leq \frac{s_{1}}{s_{2}}+\frac{s_{2}}{s_{3}}+\cdots+\frac{s_{r-2}}{s_{r-1}}+s_{r-1}-(r+1)+\frac{3}{4}
$$

(ii) if $l=r-1$ and $s_{i}$ divides $s_{i-1}$ for any $i=2, \ldots, r-1$, then

$$
e(C) \leq \frac{s_{1}}{s_{2}}+\frac{s_{2}}{s_{3}}+\cdots+\frac{s_{r-2}}{s_{r-1}}+s_{r-1}-(r+1)
$$

and, in such case, the bound is sharp;

(iii)

$$
e(C) \leq \frac{s_{1}}{s_{2}}+\frac{2 G\left(r-1 ; s_{2}, \ldots, s_{l}\right)-2-s_{2}}{s_{2}},
$$

where $G\left(r-1 ; s_{2}, \ldots, s_{l}\right)$ denotes the maximal arithmetic genus for curves verifying the flag condition $\left(r-1 ; s_{2}, \ldots, s_{l}\right)$. Moreover, if there exists some subcanonical curve verifying the flag condition $\left(r-1 ; s_{2}, \ldots, s_{l}\right)$ and with maximal genus $G\left(r-1 ; s_{2}, \ldots, s_{l}\right)$, then the bound is sharp.

When $l=2, G\left(r-1 ; s_{2}\right)$ denotes the Castelnuovo bound for nondegenerate curves in $\mathbf{P}^{r-1}$ of degree $s_{2}$ (see also Proposition 2 below). In Remark $(i)$ below, we make explicit the condition $s_{1}>>s_{2}>>\cdots>>s_{r-1}$. Recall that a projective curve $C$ is subcanonical if $\omega_{C}=\mathcal{O}_{C}(e(C))$. For integral curves, this is equivalent to require that the equality holds in (1). For instance, a Castelnuovo curve in $\mathbf{P}^{r-1}$ of degree $s_{2} \equiv 2$ $\bmod (r-2)$ is subcanonical. This gives an example for (iii). See also Proposition 2 below for further examples for flag conditions of length $\leq 3$, and Remark $(i v)$ below for a reformulation of property $($ iii).

We will see that Proposition 1 is an easy consequence of results contained in [CCD2] and $[\mathrm{D}]$. Notice that the bound $(i i)$ is sharp because it is attained by complete intersection curves of type $\left(s_{r-1}, \frac{s_{r-2}}{s_{r-1}}, \ldots, \frac{s_{1}}{s_{2}}\right)$. One expects that the bound $(i i)$ holds true in any case, i.e. without the assumption $s_{1}>>s_{2}>>\cdots>>s_{r-1} \geq 2$, and that $s_{i}$ divides $s_{i-1}$ for any $i=2, \ldots, r-1$. Also, one expects that the bound $(i i)$ it is attained only by complete intersection curves. We are not able to prove a speciality theorem in this generality, neither to eliminate the positive term $\frac{3}{4}$ appearing in $(i)$ of Proposition 1 (however we note that, by a suitable choice of the numerical assumptions on the $s_{i}$ 's, one may substitute the term $\frac{3}{4}$ with any positive number (compare with [CCD2], Remark 1.3)). Notice also that property $(i)$ of Proposition 1 improves the bound in [CCD2], Proposition 2.4, ii). 
Finally we recall that, extending previous results of Eisenbud and Harris $[\mathrm{EH}]$, in [CCD3] one generalizes Halphen bound also for the genus of curves in $\mathbf{P}^{r}$ of given degree $d$ and not contained in surfaces of degree less than a fixed number $s$. Consequently, as we said before for Theorem A, by (1) one easily obtains a corresponding bound for the speciality. Therefore, in the case of flag conditions of length 2 (i.e. $l=2$ ), one has a more precise result than previous Proposition 1. In fact, fix integers $r, d$ and $s$, with $s \geq r-1 \geq 2$, and define $m, \epsilon, w$ and $v$ by dividing $d-1=m s+\epsilon, 0 \leq \epsilon \leq s-1$, and $s-1=w(r-2)+v, 0 \leq v \leq r-2$. Denote by $G(r ; d, s)$ the maximal arithmetic genus for an integral nondegenerate projective curve $C \subset \mathbf{P}^{r}$ of degree $d$, not contained in any surface of degree $<s$. One may prove that, when $d>>s$, then

$$
G(r ; d, s)=\frac{d^{2}}{2 s}+\frac{d}{2 s}[2 G(r-1 ; s)-2-s]+R,
$$

where $G(r-1 ; s)=\left(\begin{array}{c}w \\ 2\end{array}\right)(r-2)+w v$ is the Castelnuovo bound for a nondegenerate curve of degree $s$ in $\mathbf{P}^{r-1}$, and

$$
R=\frac{1+\epsilon}{2 s}(s+1-\epsilon-2 G(r-1 ; s))+w(\epsilon-\delta)-k\left(\begin{array}{c}
w+1 \\
2
\end{array}\right)+\left(\begin{array}{l}
\delta \\
2
\end{array}\right) .
$$

The numbers $k$ and $\delta$ are defined by dividing $\epsilon=k w+\delta, 0 \leq \delta<w$, when $\epsilon<w(r-1-v)$. Otherwise define $k$ and $\delta$ by dividing $\epsilon+r-2-v=(w+1) k+\delta, 0 \leq \delta<w+1$ (compare with [CCD3]). Taking into account (1) and the previous expression for $G(r ; d, s)$, with an elementary numerical analysis based on [CCD3] we are able to prove the following:

Proposition 2. Fix integers $r$, $d$ and $s$, with $s \geq r-1 \geq 2$, and $d>>s$. Let $C \subset \mathbf{P}^{r}$ be an integral nondegenerate projective curve of degree $d$, not contained in any surface of degree $<s$. Define $m, \epsilon, w$ and $v$ by dividing $d-1=m s+\epsilon, 0 \leq \epsilon \leq s-1$, and $s-1=w(r-2)+v, 0 \leq v \leq r-2$. Put $G(r-1 ; s)=\left(\begin{array}{c}w \\ 2\end{array}\right)(r-2)+w v$. Then one has

$$
e(C) \leq \frac{d}{s}+\frac{2 G(r-1 ; s)-2-s}{s}
$$

Moreover the following properties are equivalent:

(i) $e(C)=\frac{d}{s}+\frac{2 G(r-1 ; s)-2-s}{s}$;

(ii) $p_{a}(C)=G(r ; d, s)$, and $v=0$ and $\epsilon=w$, or $v \geq 1$ and $\epsilon=w(r-1-v)+1$;

(iii) $p_{a}(C)=G(r ; d, s)$ and $C$ is subcanonical.

As before, in Remark $(i)$ below we make explicit the condition $d>>s$.

Now we are going to prove the announced results. We begin with the proof of Theorem B.

To this purpose, first we examine the case $u \geq 2$, i.e. we assume that $C \subset \mathbf{P}^{5}$ is nondegenerate. 
Assume also that

$$
e(C) \geq \frac{d}{s}+\frac{s}{t}+\frac{t}{u}+u-6
$$

Then from (1) we deduce that

$$
p_{a}(C) \geq \frac{d^{2}}{2 s}+\frac{d}{2}\left[\frac{s}{t}+\frac{t}{u}+u-6\right]+1 \text {. }
$$

On the other hand, since $C$ satisfies the flag condition $(5 ; d, s, t, u)$ and $d>>s>t>>$ $u$, from [CCD2], Proposition 1.1 and Theorem 2.2, we know that

$$
p_{a}(C) \leq G^{h}(5 ; d, s, t, u):=\frac{d^{2}}{2 s}+\frac{d}{2}\left[\frac{s}{t}+\frac{t}{u}+u-6+\eta\right]+\rho+1
$$

where $\eta$ and $\rho$ are suitable rational numbers such that

$$
|\eta| \leq \frac{3}{4} \quad \text { and } \quad|\rho| \leq 33 \frac{s^{3}}{t^{2}}
$$

From (7) and our hypotheses $d>>s>>t>>u$, we get

$$
G^{h}(5 ; d, s, t, u)-p_{a}(C) \leq \frac{d}{2} \eta+\rho<\frac{d}{2}\left[\frac{t}{u(u+1)}-3 u\right]
$$

Therefore we may apply [CCD2], Proposition 1.4 and Theorem 2.2, ii), and deduce the existence of a flag such as (2).

Our next step is to prove that $S$ and $T$ are in fact complete intersections. To this aim, we are going to analyze the sectional arithmetic genus $\pi$ of $S$ and to compare it with the quoted bound for the genus of curves verifying the flag condition $(4 ; s, t, u)$ in $\mathbf{P}^{4}$, appearing in $[\mathrm{CCD}]$.

First we need the following lemma. For its proof we refer to [D], Lemma.

Lemma 1. Let $S \subset \mathbf{P}^{r}$ be an integral, nondegenerate projective surface, of degree $s \geq r-1 \geq 2$. Denote by $\pi$ the sectional arithmetic genus of $S$. Let $C \subset S$ be an integral, nondegenerate projective curve, of degree $d \geq s^{2}$. Denote by $p_{a}(C)$ the arithmetic genus of $C$. Then one has:

$$
p_{a}(C) \leq \frac{d^{2}}{2 s}+\frac{d}{2 s}(2 \pi-2-s)+s^{3} /(r-2) .
$$

In particular, when $d>2 s^{4} / r-2$, one has

$$
e(C) \leq \frac{d}{s}+\frac{2 \pi-2-s}{s}
$$


Comparing (9) with (6) we deduce that

$$
\pi \geq \frac{s^{2}}{2 t}+\frac{s}{2}\left[\frac{t}{u}+u-5\right]+1
$$

On the other hand, by $[\mathrm{CCD}]$ we know that

$$
\pi \leq G(4 ; s, t, u)
$$

where

$$
G(4 ; s, t, u):=\frac{s^{2}}{2 t}+\frac{s}{2}\left[\frac{t}{u}+u-5-\frac{(u-1-\beta)(1+\beta)(u-1)}{u t}\right]+\rho_{1}(s, t, u)+1 .
$$

In the previous expression the number $\beta$ is defined by dividing $t-1=\alpha u+\beta, 0 \leq \beta<u$. The number $\rho_{1}(s, t, u)$ is a rational number which only depends on $s, t$ and $u$. For its definition we refer to [CCD]. For the moment we only need that

$$
\left|\rho_{1}(s, t, u)+1\right| \leq t^{3} / 3
$$

Previous estimate follows from Lemma 1, taking into account that one may interpret $G(4 ; s, t, u)$ as the arithmetic genus attained by curves in $\mathbf{P}^{4}$ lying in surfaces of degree $t$, whose sectional genus is equal to $\frac{t^{2}}{2 u}+\frac{t}{2}(u-4)-\frac{(u-1-\beta)(1+\beta)(u-1)}{2 u}+1$, i.e. is equal to the Halphen bound for curves of degree $t$, not contained in surfaces of degree $<u$ in $\mathbf{P}^{3}$ (see $[\mathrm{CCD}]$ ). From (10), (11) and (12), and our assumption $s>>t$, we obtain that

$$
u-1-\beta=0
$$

i.e. $u$ divides $t$, and that $\rho_{1}(s, t, u) \geq 0$. On the other hand, from [CD], pg.2708-2709, we know that, when $u$ divides $t$, then $\rho_{1}(s, t, u) \leq 0$, and $\rho_{1}(s, t, u)=0$ if and only if $t$ divides $s$. It follows that $u$ divides $t, t$ divides $s$ and, from (10) and (11), that

$$
\pi=\frac{s^{2}}{2 t}+\frac{s}{2}\left[\frac{t}{u}+u-5\right]+1=G(4 ; s, t, u)
$$

i.e. the general hyperplane section $S^{\prime}$ of $S$ has maximal arithmetic genus $G(4 ; s, t, u)$ with respect to the flag condition $(4 ; s, t, u)$. This in turn implies, from [CCD] again, that $T$ is a complete intersection on $U$. Moreover also $S$ is a complete intersection on $T$. In fact, from the Hilbert function of $S^{\prime}$, we know that $S^{\prime}$ is contained in a space surface of degree $\frac{s}{t}$ not containing the general hyperplane section of $T$, and such a surface lifts to $\mathbf{P}^{4}$ because $S^{\prime}$ is arithmetically Cohen-Macaulay (see [CCD], Theorem and pg. 132).

At this point, taking into account (6), to conclude the proof of Theorem $\mathrm{B}$ in the case $u \geq 2$, it suffices to prove the following lemma. We will prove it below. 
Lemma 2. Let $T \subset \mathbf{P}^{r}$ be an irreducible, reduced, projective threefold of degree $t$. Assume that $T$ is arithmetically Cohen-Macaulay and subcanonical, i.e. $\omega_{T}=\mathcal{O}_{T}(k)$ for some $k \in \mathbf{Z}$. Let $S \subset T$ be an integral surface of degree $s$, complete intersection on $T$. Let $C \subset S$ be an integral curve of degree $d$. Then one has:

$$
e(C) \leq \frac{d}{s}+\frac{s}{t}+k
$$

Moreover equality holds if and only if $C$ is a complete intersection of $S$ with a hypersurface of degree $\frac{d}{s}$.

This concludes the proof of Theorem B when $u \geq 2$. When $u=1$, taking into account [GP], we may assume that $C$ is nondegenerate in $\mathbf{P}^{4}$. In this case the proof is easier because it does not need either Lemma 1 or Lemma 2. In fact, from [CCD], we know that $p_{a}(C) \leq G(4 ; d, s, t)$ (compare with (11) and (12)). Therefore, assuming (6) and using (1), the same previous analysis of the number $\rho_{1}(d, s, t)$ shows that $t$ divides $s, s$ divides $d$ and that $C$ is a complete intersection. This concludes the proof of Theorem B.

Now we are going to prove Lemma 2.

To this purpose, consider a hypersurface $P \subset \mathbf{P}^{r}$ of degree $p>>0$ containing $C$, denote by $D$ the complete intersection $S \cap P$, and denote by $R$ the residual scheme of $C$ with respect to $D$. Since $T$ is subcanonical, the local Noether sequence of liaison corresponding to $C$ and $R$ gives:

$$
0 \rightarrow \mathcal{I}_{D} \rightarrow \mathcal{I}_{R} \rightarrow \omega_{C}\left(-k-\frac{s}{t}-p\right) \rightarrow 0
$$

$(\mathcal{I}=$ ideal sheaf $)$. Since $D$ is arithmetically Cohen-Macaulay and $h^{0}\left(C, \omega_{C}(-e(C))>0\right.$, from previous sequence it follows that there exists a hypersurface $Q$ of degree $q=$ $k+\frac{s}{t}+p-e(C)$ containing $R$ and not containing $D$. The complete intersection curve $D^{\prime}=S \cap Q$ contains $R$. Set $D^{\prime}=R \cup R^{\prime}$ the corresponding, possibly algebraic, linkage. Since $\operatorname{deg}\left(R^{\prime}\right)=d-s\left(e(C)-k-\frac{s}{t}\right)$ and $\operatorname{deg}\left(R^{\prime}\right) \geq 0$, we obtain the claimed inequality. If the equality holds, then $R^{\prime}$ is empty and $R=D^{\prime}$ is a complete intersection, with $\omega_{R}=\mathcal{O}_{R}\left(k+\frac{s}{t}+q\right)$. Coming back to the first linkage, we find

$$
0 \rightarrow \mathcal{I}_{D} \rightarrow \mathcal{I}_{C} \rightarrow \mathcal{O}_{R}(q-p) \rightarrow 0
$$

Therefore there exists a hypersurface of degree $p-q$ containing $C$ and not containing $D$. Since $d=s(p-q)$, then $C$ is the complete intersection of $S$ with such a hypersurface. This concludes the proof of Lemma 2.

As for the proof of Proposition 1, we first notice that properties $(i)$ and $(i i)$ simply follow form (1) and from [CCD2], Proposition 1.1, $(v)$, and Theorem 2.2. It remains to prove property $(i i i)$. To this purpose, denote by $G\left(r ; s_{1}, \ldots, s_{l}\right)$ the maximal arithmetic 
genus among the curves verifying the flag condition $\left(r ; s_{1}, \ldots, s_{l}\right)$. Then, from $(1)$, we have

$$
e(C) \leq \frac{2 G\left(r ; s_{1}, \ldots, s_{l}\right)-2}{s_{1}} .
$$

From [D], Theorem, $(d)$, and the fact that $s_{1}>>s_{2}$, we deduce the inequality (3). In order to prove the sharpness, let $E \subset \mathbf{P}^{r-1}$ be a subcanonical curve verifying the flag condition $\left(r-1 ; s_{2}, \ldots, s_{l}\right)$ and of maximal arithmetic genus $p_{a}(E)=G\left(r-1 ; s_{2}, \ldots, s_{l}\right)$. We have $e(E)=\left(2 G\left(r-1 ; s_{2}, \ldots, s_{l}\right)-2\right) / s_{2}$. By [D], Theorem, $(a)$, we know that $E$ is arithmetically Cohen-Macaulay. Let $C$ be a complete intersection of the cone over $E$ in $\mathbf{P}^{r}$ with a general hypersurface of degree $a>>$. The curve $C$ is arithmetically Cohen-Macaulay and, by [D], Theorem, $(b)$, it verifies the flag condition $\left(r ; s_{1}, \ldots, s_{l}\right)$, where $s_{1}=a s_{2}$. We have to prove that $e(C)=a+e(E)-1$. By (3), we already know that $e(C) \leq a+e(E)-1$. On the other hand, since $E$ is arithmetically Cohen-Macaulay, then from the exact sequence:

$$
0 \rightarrow \mathcal{O}_{E}(e(E)) \rightarrow \mathcal{O}_{E}(a+e(E)-1) \rightarrow \mathcal{O}_{\Gamma} \rightarrow 0
$$

one deduces that $h_{\Gamma}(a+e(E)+1)=s_{1}-1\left(h_{\Gamma}=\right.$ Hilbert function of the general hyperplane section $\Gamma$ of $C$ ). It follows that $e(C) \geq a+e(E)-1$ : in fact, since $C$ is arithmetically Cohen-Macaulay, then its speciality index is equal to $\max \left\{i: h_{\Gamma}(i)<\right.$ $\left.s_{1}\right\}-1$. This concludes the proof of Proposition 1 .

Now we are going to prove Proposition 2.

With the same notation as in (4), a direct computation proves that $|R| \leq s^{3} /(r-$ $2)$. This also follows from Lemma 1 , because $G(r ; d, s)$ is attained by curves lying in Castelnuovo surfaces of degree $s$. Therefore, since $d>>s$ and $p_{a}(C) \leq G(r ; d, s)$, from (1) we deduce the bound (5).

Another way to obtain (5) is the following: denote by $h_{\Gamma}(i)$ the Hilbert function of the general hyperplane section $\Gamma$ of $C$, and by $h(i)$ the Hilbert function of the general hyperplane section of any nondegenerate curve of degree $d$, not contained in surfaces of degree $<s$, with maximal genus $G(r ; d, s)$. In [CCD3] one proves that this function $h(i)$ depends only on $r, d$ and $s$, and it may be explicitly computed. Since the speciality index of $C$ is $\leq \max \left\{i: h_{\Gamma}(i)<d\right\}-1$, and $h_{\Gamma}(i) \geq h(i)$ for any $i$, it follows that $e(C) \leq \max \{i: h(i)<d\}-1$. An elementary computation proves that this last number is bounded as in (5).

Now, if the equality holds in (5), then the number $e(C)=\frac{d}{s}+\frac{2 G(r-1 ; s)-2-s}{s}$ is an integer. A computation proves that this is equivalent to say that $v=0$ and $\epsilon=w$, or that $v \geq 1$ and $\epsilon=w(r-1-v)+1$. In these cases in (4) one has $R=1$, and therefore from (1) it follows that $p_{a}(C) \geq G(r ; d, s)$, hence $p_{a}(C)=G(r ; d, s)$. This proves that $(i)$ implies $(i i)$. Conversely, if property $(i i)$ holds true, then $C$ is a curve with maximal genus $G(r ; d, s)$. Then $C$ is arithmetically Cohen-Macaulay and so $e(C)=\max \{i: h(i)<d\}-1$. A computation proves that this number is just $\frac{d}{s}+\frac{2 G(r-1 ; s)-2-s}{s}$. This proves that $(i i)$ implies $(i)$. 
Moreover, if $(i i)$ holds, then as before one has $R=1$. Therefore, using also $(i)$, we obtain $e(C)=\left(2 p_{a}(C)-2\right) / d$, and so $C$ is subcanonical. This proves that $(i i)$ implies (iii). Conversely, if $p_{a}(C)=G(r ; d, s)$ and $C$ is subcanonical, then

$$
e(C)=\left(2 p_{a}(C)-2\right) / d=\frac{d}{s}+\frac{2 G(r-1 ; s)-2-s}{s}+\frac{2(R-1)}{d} .
$$

Since $|R| \leq s^{3} /(r-2)$ and $d>>s$, then previous equality implies that $R=1$, and so the equality holds in (5). This proves that (iii) implies $(i)$, and concludes the proof of Proposition 2.

Remark. ( $i$ ) In proving Theorem B, we need the numerical assumption $d>>s>>$ $t>>u$ only to use the quoted results in [CCD], [CCD2] and [D], and to prove (8), (9) and (13). To this purpose, an elementary computation proves that it suffices assume that:

$$
d>\frac{2}{3} s^{4}, \quad s>\frac{2}{3} t^{4}, \quad t>408(u+1)^{3}, \quad u \geq 2,
$$

or

$$
d>\max \left\{\frac{2}{3} s^{4}, 12(s+1)^{2}\right\}, \quad s>t^{2}-t, \quad t \geq 2, \quad u=1 .
$$

As for the proof of Proposition 1, in order to deduce $(i)$ and $(i i)$ from (1) and [CCD2], it suffices to add to the same numerical explicit assumption appearing in [CCD2], Theorem 2.2 , the request that

$$
s_{1}>2(r-l)\left(l^{2}+2 l+9\right) \frac{s_{2}^{3}}{s_{3}^{2}} s_{2} \cdot \ldots \cdot s_{l}
$$

(when $l=2$, put $s_{3}=r-3$ ). Similarly, to prove (iii) we need the same numerical explicit assumption appearing in [CCD3], Main Theorem, with the further request that

$$
s_{1}>\frac{2 s_{2}^{4}}{r-2}
$$

Also to prove Proposition 2 we need the same numerical explicit assumptions appearing in [CCD3], Main Theorem, with the further request that

$$
d>\frac{2 s^{4}}{r-2}
$$

which enables us to deduce that $R=1$ from (14).

All previous numerical hypotheses are certainly not the best possible. They only are of the simplest form we were able to conceive.

(ii) We think it would be interesting to understand extremal curves with respect inequality (9) in Lemma 1. For instance, when $S$ is a smooth and subcanonical surface, one may prove that its curves $C$ with extremal speciality are necessarily subcanonical. In fact if $C$ has speciality $e(C)=\frac{d}{s}+\frac{2 \pi-2-s}{s}$ then $p_{a}(C) \geq \frac{d^{2}}{2 s}+\frac{d}{2 s}(2 \pi-2-s)+1$. On 
the other hand, Hodge index Theorem and the assumption on $S$ imply that $p_{a}(C) \leq$ $\frac{d^{2}}{2 s}+\frac{d}{2 s}(2 \pi-2-s)+1$, and therefore $p_{a}(C)=\frac{d^{2}}{2 s}+\frac{d}{2 s}(2 \pi-2-s)+1$. It follows that $e(C)=\frac{2 p_{a}(C)-2}{d}$ and so $C$ is subcanonical.

Is this conclusion true without assuming $S$ is smooth and subcanonical ?

(iii) From Proposition 2 it follows that a curve $C$ with maximal speciality has arithmetic genus given by the formula (4) with $R=1$. We notice that this condition is not enough to ensure that the speciality is maximal. In fact, as soon as $\epsilon=s-1$, in the formula (4) one has $R=1$. Notice also that, in the particular case $s=r-1$, Proposition 2 simply says that for an integral nondegenerate curve $C \subset \mathbf{P}^{r}$ of degree $d$ one has $e(C) \leq \frac{d}{r-1}-\frac{r+1}{r-1}$, and the equality holds if and only if $C$ is a Castelnuovo curve with $d \equiv 2 \bmod (r-1)$. This is a (we think well known) consequence of Castelnuovo bound, and it holds for any $d \geq r$.

$(i v)$ In the case there exists some subcanonical curve verifying the flag condition $\left(r-1 ; s_{2}, \ldots, s_{l}\right)$ and with maximal genus $G\left(r-1 ; s_{2}, \ldots, s_{l}\right)$, then one may reformulate property (iii) of Proposition 1 as follows:

$$
e\left(r ; s_{1}, \ldots, s_{l}\right)=\frac{s_{1}}{s_{2}}+e\left(r-1 ; s_{2}, \ldots, s_{l}\right)-1
$$

where $e\left(r ; s_{1}, \ldots, s_{l}\right)$ denotes the maximal speciality index among the curves verifying the flag condition $\left(r ; s_{1}, \ldots, s_{l}\right)$.

\section{REFERENCES}

[CCD] L. Chiantini, C. Ciliberto and V. Di Gennaro, The genus of curves in $\mathbf{P}^{4}$ verifying certain flag conditions, Manuscripta Math. 88 (1995), 119-134.

[CCD2] L. Chiantini, C. Ciliberto and V. Di Gennaro, On the genus of projective curves verifying certain flag conditions, Boll. U.M.I. (7) 10-B (1996), 701-732.

[CCD3] L.Chiantini, C.Ciliberto and V.Di Gennaro, The genus of projective curves, Duke Math. J. 70/2 (1993), 229-245.

[CD] C. Ciliberto and V. Di Gennaro, Factoriality of certain threefolds complete intersection in $\mathbf{P}^{5}$ with ordinary double points, Commun. Algebra 32 (7) (2004), 2705-2710.

[D] V. Di Gennaro, Hierarchical structure of the family of curves with maximal genus verifying flag conditions, preprint, math.AG/0504576 (2005).

[EH] D. Eisenbud and J. Harris, Curves in Projective Space, Sém. Math. Sup. 85, Les Presses du l'Université de Montréal, Montréal (1982).

[GP] L. Gruson and C. Peskine, Genre des courbes dans l'espace projectif, Algebraic Geometry: Proceedings, Norway, 1977, Lecture Notes in Math., Springer-Verlag, New York 687 (1978), $31-59$. 